\title{
INTERACTION OF DISKS AND DARK HALOES OF DWARF SPIRALS
}

\author{
B. FUCHS, V. FRIESE, H. REFFERT, R. WIELEN \\ Astronomisches Rechen - Institut Heidelberg \\ Mönchhofstr. 12 - 14, 69120 Heidelberg, Germany
}

\section{Introduction}

In a number of dwarf spiral galaxies the HI-emission has been studied with sufficient resolution to derive the rotation curves of the galaxies. These show that the disks of dwarf spirals are imbedded in extended haloes of dark matter, quite similar to the disks of giant spiral galaxies.

The presence of such massive, extended haloes affects the dynamics of the disks of the galaxies. We concentrate on two aspects here: First we discuss the effects of large amounts of dark matter on the spiral structure of the disks. Unfortunately the velocity dispersion of the stars in dwarf spirals is not known, but we have to use the vertical scale heights of the disks to derive estimates of the velocity dispersions. Once the velocity dispersions are known Toomre's $\mathrm{Q}$ stability parameter and ' $\mathrm{X}=2$ ' criterion (Toomre 1964, 1981, Athanassoula et al.1987) can be evaluated.

Second, it has been suggested that the main constituents of the dark haloes of giant spiral galaxies might be very compact objects such as black holes with masses of the order of a few $10^{6} \mathcal{M}_{\odot}$ (Lacey and Ostriker 1985). This scenario has been put forward among others in order to explain the observed increase of the velocity dispersion of the stars in the disk of the Galaxy (Wielen 1977). We have discussed the scattering of stars by massive black holes penetrating the disk in some detail elsewhere and found many attractive features, fitting nearly ideally the locally or globally observed kinematics of the stars in the Galaxy (Wielen et al. 1992). Thus it is interesting to carry this conjecture over to the haloes of dwarf spiral galaxies, especially since the disks of these galaxies are much less massive and thus more fragile than in giant spirals. 


\section{Spiral Structure}

We have analyzed in detail the disks of a set of 11 dwarf spirals listed in table 1 for which rotation curves are available in the literature, mainly by Carignan and collaborators (cf. Côté et al. 1991 and references therein). Dwarf spirals have no bulge component (Freeman 1987). So a mass model comprising an exponential disk and a dark halo component described by a pseudo isothermal density law has been fitted to the rotation curve of each galaxy. We discuss usually a best $\chi^{2}$-fit and a 'maximum disk' fit. Next, the vertical velocity dipersions of the stars are estimated from the vertical scale height of each disk using the vertical hydrostatic equilibrium condition. The vertical velocity dispersions are then converted to radial velocity dispersions by adopting the same axial ratio of the velocity ellipsoid as in the Milky Way. Since even the vertical scale heights of the galaxies in table 1 are not known we estimate the vertical scale heights from the radial scale lengths. For this purpose we have performed a statistical flattening analysis of faint dwarf spirals in the ESO-Uppsala catalogue in order to derive the intrinsic ratio of vertical to radial exponential scale lengths $\mathrm{z}_{0} / \mathrm{h}$. From a sample of 115 galaxies with $M_{B} \geq-18$ and Hubble type Sc or later we find $z_{0} / \mathrm{h}=$ $0.2 \pm 0.05$ corresponding to an intrinsic flattening $q_{0}=0.14$.

In this way we have modelled individually the disk of each galaxy. There is a clear distinction between the dynamical states of galactic disks which develop ordered spiral structure or which appear irregular. NGC 300 is a typical example of the first class. The stability parameter is in the range where swing amplification is effective. The expected number of spiral arms, estimated using the ' $\mathrm{X}=2$ ' criterion, indicates a two-armed pattern in the inner parts of the disk which breaks up in filaments in the outer parts, exactly as observed. NGC 55 is a typical irregular galaxy. The values of the stability parameter are so large that any spiral structure is suppressed. The transition from Hubble types Sc, Sd to Sm, Im is correlated, as can be seen from table 1, to the ratio of disk to dark halo mass within one Holmberg radius. Although our dynamical models rely on rather crude estimates of the stellar velocity dispersions and ambiguous decompositions of the rotation curves our conclusions appear to be rather robust, because we could interpret the Hubble type of each galaxy consistently.

\section{Disk Heating}

Assuming now that the stochastic heating of the disks is due to the black holes of the dark haloes, we can deduce from the velocity dispersions of the stars typical black hole masses. Wielen (1977) has determined empirically the diffusion coefficient, which describes the increase of the velocity dispersion of stars in the solar neighbourhood. Lacey and Ostriker (1985) have 
TABLE 1.

\begin{tabular}{lcccccc}
\hline & type & $M_{B}$ & $\begin{array}{c}\mathrm{M}_{d}^{\dagger} \\
\text { mag }\end{array}$ & $\begin{array}{c}\mathrm{M}_{h}{ }^{8} \mathcal{M}_{\odot}\left(\mathrm{r}<\mathrm{R}_{\text {Holm }}\right) \\
10^{8} \mathcal{M}_{\odot}\end{array}$ & $\mathrm{M}_{d} / \mathrm{M}_{h}$ & $\begin{array}{c}\mathrm{m}_{h} \\
10^{6} \mathcal{M}_{\odot}\end{array}$ \\
\hline UGC 2259 & Scd & -16.5 & $31(41)$ & $30(15)$ & $1(3)$ & $2(40)$ \\
NGC 247 & Sd & -18.0 & $87(102)$ & $121(79)$ & $0.7(1.3)$ & $12(81)$ \\
NGC 300 & Sd & -17.9 & $35(52)$ & $67(41)$ & $0.5(1.3)$ & $1.6(7)$ \\
NGC 1560 & Sd & -16.4 & $14(17)$ & $29(28)$ & $0.5(0.6)$ & $1.2(1.9)$ \\
NGC 7793 & Sd & -18.3 & $35(-)$ & $106(-)$ & $0.3(-)$ & $0.4(-)$ \\
\hline DDO 168 & IBm & -15.2 & $2(4)$ & $6(4)$ & $0.3(1)$ & $0.05(0.17)$ \\
DDO 170 & Irr & -15.2 & $1.4(2.7)$ & $9.4(7.7)$ & $0.2(0.4)$ & $0.06(0.2)$ \\
NGC 5585 & Sd & -17.5 & $7.4(14)$ & $108(99)$ & $0.07(0.14)$ & $0.04(0.1)$ \\
DDO 154 & Im & -13.8 & $-(0.28)^{*}$ & $-(2.9)$ & $-(0.1)$ & - \\
NGC 55 & Sm & -18.6 & $3(14)^{*}$ & $170(159)$ & $0.02(0.09)$ & $-(0.07)$ \\
NGC 3109 & Sm & -16.8 & $-(1.8)^{*}$ & $-(56)$ & $-(0.03)$ & - \\
\hline
\end{tabular}

${ }^{\dagger}$ Maximum disk estimates given in parantheses. * HI disk mass not included.

calculated the diffusion coefficient theoretically and shown that it is proportional to the space density times the individual mass of the black holes. This implies a mass of $3 \cdot 10^{6} \mathcal{M}_{\odot}$ of a typical black hole in the Galaxy. Quite a similar value of $2 \cdot 10^{6} \mathcal{M}_{\odot}$ can be derived for NGC 3198, another giant Sc galaxy $\left(M_{B}=-19.4\right)$, using the the disk and dark halo parameters of Bottema (1988) and van Albada et al.(1985). The typical black hole masses, which we find for the Sd galaxies are of the same order of magnitude as in giant spirals. The velocity dispersions of the disk stars of the irregular galaxies, however, are hardly larger than the turbulent velocity dispersion of the interstellar gas, so that the disks of these galaxies cannot have experienced much stochastic heating. Consequently, the masses of the hypothetical black holes in these galaxies turn out to be low, of the order of $10^{4}$ to $10^{5} \mathcal{M}_{\odot}$. This was noted before by Rix and Lake (1993) and Fuchs and Wielen (1993). Such low masses contradict the supposed universal nature of the black holes and cast severe doubts on the scenario of dark haloes of galaxies made of massive black holes.

Furthermore, if the dark haloes of the irregular galaxies were made up of black holes with 'standard' masses of a few $10^{6} \mathcal{M}_{\odot}$, the two-bodyrelaxation time of such a system would be much shorter than a Hubble time. In a galaxy like DDO 154 the entire dark halo would consist of about only 1000 black holes. We have run several N-body simulations which show that the ensemble of black holes evolves very fast within a Hubble time, 
developing a concentrated core of a few objects and a diluted halo expanded to $100 \mathrm{kpc}$ scale.

\section{References}

van Albada, T.S., Bahcall, J.N., Begeman, K., \& Sancisi, R. 1985, ApJ 295, 305

Athanassoula, E., Bosma, A., \& Papaioaunou, S. 1987, A\&A 179, 23

Bottema, R. 1988, A\&A 197, 105

Côté, S., Carignan, C., \& Sancisi, R. 1991, AJ 102, 904

Freeman, K. C. 1987, IAU Symp. No. 117, p.119

Fuchs, B., \& Wielen, R. 1993, AG Abstract Ser. 8, 145

Lacey, C., \& Ostriker, J. P. 1985, ApJ 299, 633

Rix, H. W., \& Lake, G. 1993, ApJ 417, L1

Toomre, A. 1964, ApJ 139, 1214

Toomre, A. 1981, in The Structure and Evolution of Normal Galaxies, eds. S. M. Fall \& D. Lynden-Bell (Cambridge: Cambridge Univ. Press) p.111

Wielen, R. 1977, A\&A 60, 263

Wielen, R., \& Fuchs, B. 1983, in Kinematics, Dynamics and Structure of the Milky Way, ed W. L. H. Shuter (Dordrecht: Reidel) p.81

Wielen, R., Dettbarn, C., Fuchs, B., Jahreiß, H., \& Radons, G. 1992, IAU Symp. No. 149, p.81

\section{Discussion}

Kormendy: Your point that the dynamical timescale for a DM halo made of $100 \cdot 10^{6} \mathcal{M}_{\odot} \mathrm{BHs}$ is short, is well taken and can be strengthened still more by considering the lowest luminosity dwarf spheroidal galaxies. They have halo masses that are so small that only a few $-10 \mathrm{BHs}$ would be required. Then the dynamical timescale for halo evolution and galaxy evaporation is extremely short - only a modest number of crossing times. I have felt for some years that this is one of the strongest arguments against the idea that DM halos are made of $10^{6} \mathcal{M}_{\odot}$ BHs.

Moore: Moore (1994, ApJ 413, L93) and Moore \& Silk (1995, ApJ 442, L5) use the existence of low luminosity globular clusters and the invariance with position of the globular cluster luminosity function, to constrain the mass of possible dark matter candidates in galactic halos. Our limits are of the order of $10^{4}$ to $10^{5} \mathcal{M}_{\odot}$.

McGaugh: $\mathrm{M}_{H}\left(\mathrm{r}<\mathrm{R}_{*}\right) / \mathrm{M}_{\text {disk }}$ is strongly correlated with L. At fixed L, it is strongly correlated with surface brightness. Understanding these correlations is fundamental to the problem of galaxy formatiom and evolution in dark matter halos. Is there any reason to expect these correlations in either baryonic or non-baryonic dark matter scenarios?

Fuchs: One might add that in irregular galaxies the dark haloes tend to be more concentrated and have higher central densities than in Sd galaxies. However, these correlations do not seem to be related to the present day dynamics of the galaxies but to the formation processes of the galaxies. 